\title{
Secretory symptoms from metastatic adrenal cortical carcinoma responding to octreotide
}

\author{
HS Pandha, K Harrington, S Saini, J Lynn, M Peters, J Waxman
}

\begin{abstract}
Summary
The prognosis of patients with metastatic adrenal cortical carcinoma is poor, and their disabling symptoms are usually unresponsive to conventional therapy. $A$ patient with Cushing's syndrome secondary to a secretory adrenal cortical carcinoma was treated with octreotide, endocrine therapy and chemotherapy having failed. Treatment led to a dramatic relief of her symptoms with a fall in corticosteroid secretion. Somatostatin analogue therapy for this tumour should be encouraged in view of the lack of alternative palliative treatment.
\end{abstract}

Keywords: octreotide, adrenal cortex, carcinoma

\section{Introduction}

Adrenal cortical carcinoma is a rare cause of Cushing's syndrome. At least half of all patients have metastatic disease at presentation. No adjuvant treatment is effective, and relapse is followed by a median survival of approximately three months. There is no effective cytotoxic drug or drug combination available for recurrent adrenal cortical carcinoma; $30-40 \%$ of patients will have a partial response to treatment, but the median survival is four months. ${ }^{1}$ The management of locally recurrent or metastatic tumour is therefore aimed at palliating symptoms secondary to hormonal hypersecretion, visceral pain, or bone pain. Agents such as mitotane and aminoglutethimide given to reduce hypercortisolaemia are relatively ineffective and are themselves toxic. There is a need for new treatments of this condition that are not toxic and which will palliate hypersecretory symptoms. For this reason we have explored the value of octreotide in adrenal cortical carcinoma.

Department of

Clinical Oncology,

Hammersmith

Hospital,

Du Cane Road, London W12 0NN, UK

HS Pandha

K Harrington

S Saini

J Lynn

M Peters

J Waxman

Correspondence to

Dr J Waxman

Accepted 27 September 1994

\section{Case report}

A 46-year-old woman presented in May 1993 with weight gain, amenorrhoea, proximal myopathy, and severe acne. She had many of the clinical stigmata of Cushing's syndrome and a right-sided abdominal mass. Her initial greater than 3000 (normal range $0-270 \mathrm{nmol}$ / $24 \mathrm{~h}$ ) and her morning plasma cortisol level was over 800 (normal range $200-700 \mathrm{nmol} / \mathrm{l}$ ) with sion of plasma or UFCs by high-dose dexamethasone. Adrenocorticotrophic hormone (ACTH) levels were undetectable. There was marked hypokalaemic alkalosis and a raised serum testosterone level of 5.9 (normal range 0-3 nmol/1). Imaging investigations showed a large right-sided adrenal tumour without metastases.

A right adrenalectomy was performed and a large tumour weighing $585 \mathrm{~g}$ and measuring $12 \times 12 \times 7.5 \mathrm{~cm}$ was resected. Histology showed invasion of the pseudocapsule by an adrenal cortical carcinoma, with infiltration of a para-aortic lymph node and the inferior vena cava.

She made an excellent recovery from surgery with rapid resolution of all symptoms, although a short synacthen test four months later still showed some evidence of adrenal suppression. Serial 24-h UFCs were normal. Over the next six weeks her symptoms returned. Her 24-h UFCs were once again raised to over $3200 \mathrm{nmol}$, and restaging showed local recurrence, para-aortic lymphadenopathy, and pulmonary and liver metastases.

Over a period of three months she had symptomatic and radiological evidence of disease progression, uncontrolled by treatment with mitotane and aminoglutethimide which were both poorly tolerated. There was further disease progression despite metyrapone (500 mg, tid) and cisplatin $100 \mathrm{mg} / \mathrm{m}^{2}$. At this point treatment was initiated with octreotide $300 \mu \mathrm{g}$ subcutaneously tid in an attempt to palliate her secretory symptoms. A pretreatment Octreoscan ([11' $\left.\ln -\mathrm{DTPA}-\mathrm{D}-\mathrm{Phe}^{1}\right]$ octreotide, Mallinkrodt Diagnostica, Petten, Holland) was negative for somatostatin receptors and showed reduced uptake in the metastases. Her symptoms improved rapidly with octreotide with an accompanying reduction in UFCs but not serum levels of testosterone or dihyroepiandosterone sulphate (figure). The improvement was sustained, enabling the patient to return to her home overseas. 24-hour urinary free cortisols (UFCs) were loss of diurnal variation, but without suppres-

\section{Comment}

Octreotide is effective at controlling endocrine hypersecretion and hormone-induced symptoms associated with a variety of functional endocrine tumours. ${ }^{2-3}$ In view of the lack of therapeutic options for adrenal cortical carcinoma and the broad spectrum of inhibitory endocrine effects of somatostatin analogues, octreotide was successfully applied as treat- 


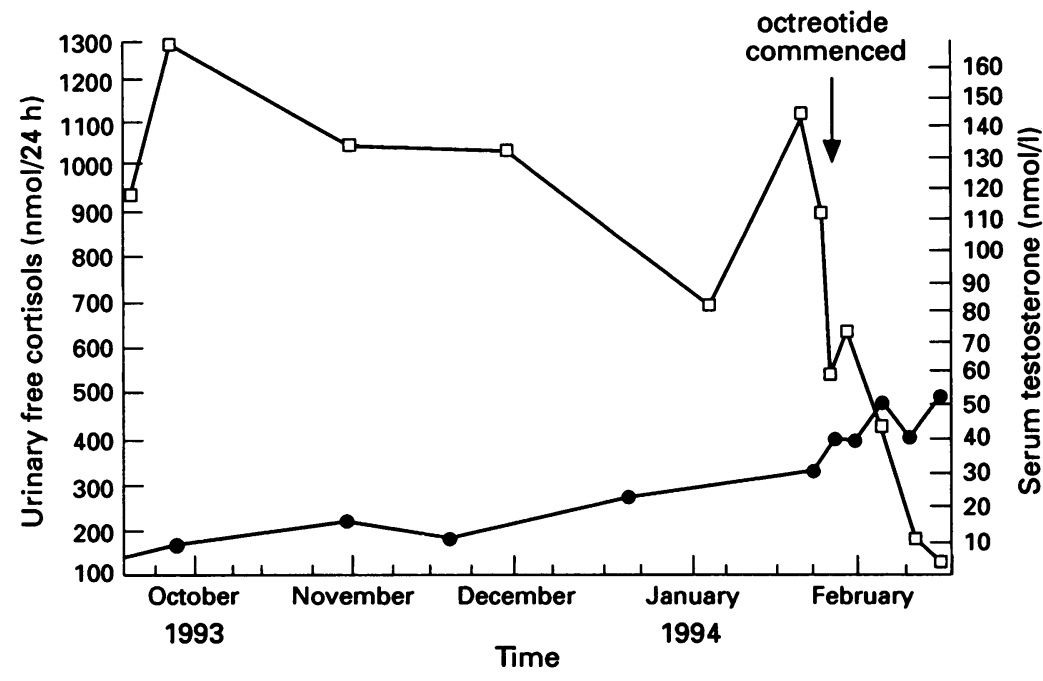

Figure Profile of urinary free cortisols and serum testosterone. $\square=$ UFCs; $=$ serum testosterone
Indications for octreotide therapy

- functional endocrine tumours

- hypercalcaemia

- interstinal obstruction

to inhibit aldosterone secretion. A marked decrease of plasma cortisol levels with infusional somatostatin was observed by Aynsley-Green et al in an evaluation of the influence of this peptide on insulin secretion. ${ }^{5}$ Octreotide has also been shown to reduce UFCs in patients with ACTH-dependent Cushing's syndrome, ${ }^{6}$ but the primary site of action of somatostatin could not be inferred from any of these studies.

In our patient, despite the negative Octreoscan, there was a rapid symptomatic and biochemical improvement in response to octreotide. The mechanism for this is unknown. The response to octreotide in ACTHindependent adrenal cell carcinoma demonstrated by the serial measurement of UFCs has not previously been reported. In view of the limited efficacy of current agents in adrenal cortical carcinoma, a trial of octreotide to control secretory symptoms in patients with this condition would seem reasonable.

We thank Dr J Lambert and Dr R Scott for referring the patient.

5 Aynsley-Green A, Barnes ND, Adrian TE, Kingston J, Bloom SR. Effect of somatostatin infusion on intermediate metabolism and entero-insular hormone release in infants with hyperinsulinaemic hypoglycaemia. Acta Paediatr Scand 1981; 70: 889-95.

1 Icard P, Chapuis Y, Andresian B, Bernard A, Proy C. Adrenocortical carcinoma in surgically treated patients: a retrospective study by the French As

2 Khoo D, Riley J, Waxman J. Control of emesis in bowel obstruction in terminally ill patients. Lancet 1992; 339: $375-6$.

3 Harrison M, James N, Broadley $\mathrm{K}$, et al. Somatostatin analogue treatment for malignant hypercalcaemia. $B M$ 1990; 300: 1313-4.

4 Invitti C, De Martin M, Bunam M, Cavagini F. Treatment 201-99.5 (Sandostatin). Clin Endocrinol 1990; 32: 275-81.
6 Lamberts SWJ, Uitterlinden P, Klijn SMG. The effect of long-acting somatostatin analogue SMS 201-995 on ACTH secretion in Nelson's syndrome and Cushing's disease. Acto Endocrinol 1989; 120: 760-6. 